\title{
ChemComm
}

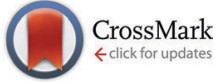

Cite this: Chem. Commun., 2015, 51,11100

Received 4th May 2015, Accepted 8th June 2015

DOI: $10.1039 / c 5 c c 03714 j$

www.rsc.org/chemcomm

\section{Giant tubular and toroidal vesicles from self-assembled triblock copolymer-polyaniline complexes in water $\dagger$}

\author{
Anbazhagan Palanisamy and Qipeng Guo*
}

\begin{abstract}
An ABA type amphiphilic triblock copolymer was synthesized via ATRP and sulfonation. New self-assembled morphologies such as toroidal vesicles, giant tubular vesicles, and perforated spherical vesicles were observed from triblock copolymer-polyaniline complexes in water. The mechanism of morphology transformation at different compositions was discussed.
\end{abstract}

The bilayers forming amphiphilic block copolymers self-assemble into a variety of vesicular morphologies such as vesicles and tubes in aqueous solution. ${ }^{1,2}$ Due to the potential application of these nanostructures in biomimicry and nanomedicine, research has been focussed on developing bilayer nanostructures of multiple geometries. ${ }^{3}$ Apart from the common bilayer structure such as spherical vesicle, complex nanostructures such as disks ${ }^{4}$ and compartmental vesicles ${ }^{5}$ were identified in recent years. The geometric complexity among self-assembled block copolymer nanostructures was achieved by either synthesizing tailored block copolymers or adding additives to block copolymers to form blends. ${ }^{6}$ Even though modern polymer synthetic techniques offer excellent control over polymer architecture and functionality, polymer blending-complexation has advantages in its own right both in solution and bulk. ${ }^{7}$ The complexation between the components was generally brought via hydrogen bonding, coordination and acidbase complexation. ${ }^{8-10}$ A variety of self-assembled morphologies has been reported from block copolymer-homopolymer complexes in aqueous solution. For instance, Gohy et $a .^{11}$ have studied a number of block copolymer-homopolymer systems where micellar aggregates are observed with tuneable morphologies. Preparing polymer aggregates through polymer complexation eliminates the need to synthesize tailored polymers to achieve specific morphology and provides flexibility for morphology tuning by taking control of the electrostatic interactions.

Polymers Research Group, Institute for Frontier Materials, Deakin University, Locked Bag 20000, Geelong, Victoria 3220, Australia.E-mail:qguo@deakin.edu.au $\dagger$ Electronic supplementary information (ESI) available: Triblock copolymer synthesis and characterization, complex preparation and FTIR analysis of complexes were elaborated in this section. See DOI: $10.1039 / \mathrm{c} 5 \mathrm{cc} 03714 \mathrm{j}$
In aqueous self-assembly of amphiphilic block copolymers, depending on required morphology, additives can be added that form complex with either wall ${ }^{12}$ or corona $^{13}$ forming blocks. The additives range from small molecules to block copolymers through metal ions and homopolymers. ${ }^{14-16}$ As the result of complexation of flexible chain block copolymer and homopolymer additives, complex morphologies such as compartmentalized micellar shells and cores has been reported in previous studies. ${ }^{14}$ However, complexation of flexible block copolymers with semi-flexible or rigid rod-like polymer additives and their self-assembly have not been studied in detail. In natural systems, the cells are made of a mixture of natural polymers that are held together by non-covalent interactions and have varying rigidity throughout the system brought by varying compositions. ${ }^{17}$ In the same vein, understanding the self-assembly mechanism and complexation of rigid polymer complexes may give new insights towards achieving biomimicry.

In this study, we have used a rigid rod-like homopolymer polyaniline (PANI) to form complex with an amphiphilic triblock copolymer polystyrene sulfonic acid- $b$-polyethylene oxide$b$-polystyrene sulfonic acid ([PSS-PS]-PEO-[PS-PSS]) and studied their self-assembled morphologies at different concentrations of PANI. Since PANI forms complex with the bilayer forming hydrophobic polystyrene blocks, small change in PANI concentration was expected to produce a pronounced effect on the final morphologies. Morphologies of aggregates formed from non-sulfonated block copolymer polystyrene- $b$-polyethylene oxide- $b$-polystyrene (PS-PEO-PS) were also studied for comparison. Fig. 1 shows the complexation between sulfonic acid groups $\left(-\mathrm{SO}_{3} \mathrm{H}\right)$ of [PSS-PS]PEO-[PS-PSS] and imine groups $(=\mathrm{N}-)$ of PANI. [PSS-PS]-PEO[PS-PSS] synthesis, characterization, complex preparation and FTIR analysis of complexes were elaborated in the ESI. $\dagger$ The selfassembled aggregates were prepared at various molar ratios of aniline to styrene sulfonic acid groups expressed as [ANI]/[SSA] ranging from 0.1 to 1.5 . The morphology of the aggregates were studied using transmission electron microscopy (TEM, JEOL JEM-2100), scanning electron microscopy (SEM, Zeiss Supra $55 \mathrm{VP}$ ) and dynamic light scattering (DLS, Zetasizer Nano ZS) techniques. 


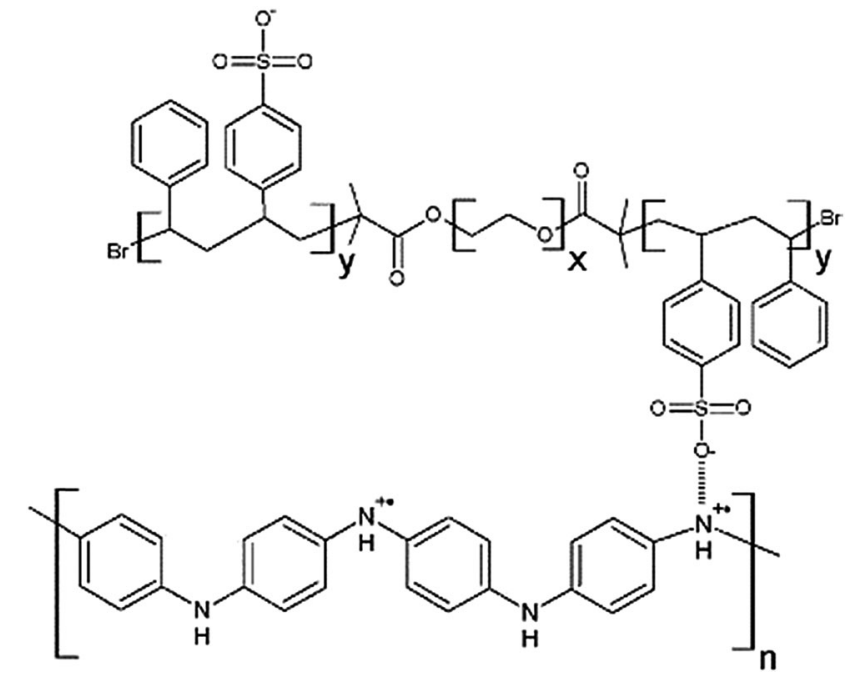

Fig. 1 Schematic representation of complexation between [PSS-PS]PEO-[PS-PSS] and PANI.

In non-sulfonated triblock copolymer-polyaniline system, PS-PEO-PS/PANI, the vesicles were the only morphology observed at all PANI concentrations ranging from 0.1 to 1.5. The TEM images of morphologies corresponding to [ANI]/[EO] ratios 0, 0.5 and 1.0 were shown in Fig. 2. For the pure PS-PEO-PS, spherical vesicles can be seen in Fig. 2a with polystyrene blocks forming the wall of the vesicle observed as dark ring due to ruthenium tetroxide staining. As PANI was added, the vesicles started forming macroscopic aggregates without change in their morphology (Fig. 2b and c) and this can be evidenced from the DLS peaks (Fig. 3) showing very broad size distribution at $[\mathrm{ANI}] /[\mathrm{EO}]=$ 0.5 and 1.0. Since PANI cannot form complex with either of hydrophilic PEO or hydrophobic PS blocks, the morphology of

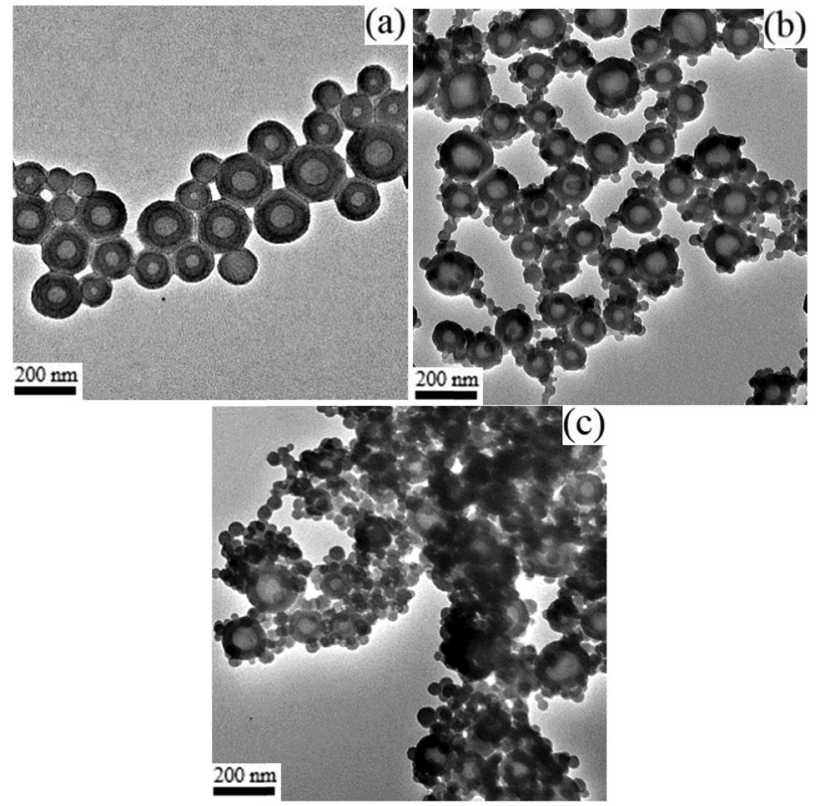

Fig. 2 TEM images of PS-PEO-PS/PANI aggregates at $[A N I] /[E O]=0$ (a), 0.5 (b) and 1.0 (c).

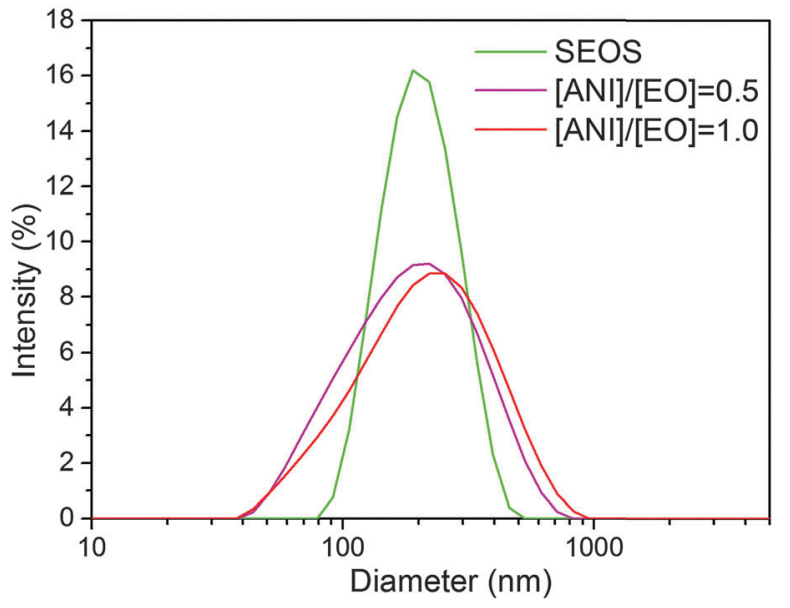

Fig. 3 Hydrodynamic diameter $\left(D_{h}\right)$ of vesicles from PS-PEO-PS/PANI blends in water.

the vesicles remained unaffected upon adding PANI. Even though hydrogen bonding between PEO and PANI was reported in the bulk state, complexation in aqueous solution was not observed here, which may be due to strong hydrogen bonding between PEO and water molecules prevented PANI complexation. ${ }^{18}$ These free PANI chains formed small spherical aggregates or precipitates in addition to vesicles (Fig. 2b and c). A similar behaviour was observed in another study, where excessive free PANI formed PANI clusters. ${ }^{19}$ In DLS study, for pure PS-PEO-PS block copolymer, a narrow DLS peak was observed at $211 \mathrm{~nm}$ indicating a monodisperse population of vesicles. However, at polyaniline concentrations above 0.3 , broad DLS peaks were observed at $217([\mathrm{ANI}] /[\mathrm{EO}]=0.5)$ and $246 \mathrm{~nm}([\mathrm{ANI}] /$ $[\mathrm{EO}]=0.1$ ) indicating polydispersity (Fig. 3). Polydispersity may be due to aggregation of phase separated PANI homopolymer, which eventually lead to macroscopic precipitation of vesicles. To enable PANI complexation and solubility, PS blocks of PS-PEO-PS were functionalized with sulfonic acid and studied the morphology of complex aggregates at various PANI concentrations.

In sulfonated triblock copolymer-polyaniline system, [PSS-PS]PEO-[PS-PSS]/PANI, morphology transformation of the vesicles was observed upon increasing PANI concentration. Fig. 4 and 5 shows the TEM and SEM images of various morphologies observed at $[\mathrm{ANI}] /[\mathrm{SSA}]=0,0.4,0.7$ and 0.9 . The sulfonated triblock copolymer self-assembled into spherical vesicles (Fig. 4a and b) of hydrodynamic diameter $\left(D_{\mathrm{h}}\right) 222 \mathrm{~nm}$ (Fig. 6). After sulfonation, the increase in size of vesicles may be due to electrostatic repulsion among negatively charged coronal chains. The water soluble polystyrene sulphonic acid groups are expected to reside in the PS/PEO interface forming a shell around PS bilayer. Fig. 4c and d shows the TEM and SEM image of toroidal vesicles, respectively, at [ANI]/ $[\mathrm{SSA}]=0.4$. Bulky toroids ${ }^{20}$ and toroidal micelles ${ }^{21}$ have been reported previously. However, toroidal vesicles have been observed for the first time in this study from block copolymer complexes. According to Eisenberg et al., ${ }^{22}$ the core chain stretching, coresolvent interaction and intercoronal repulsion are the factors that govern self-assembled morphology in block copolymer systems. Here, complexation of PANI to PSS blocks affected these selfassembly parameters and led to the morphology transformation. 


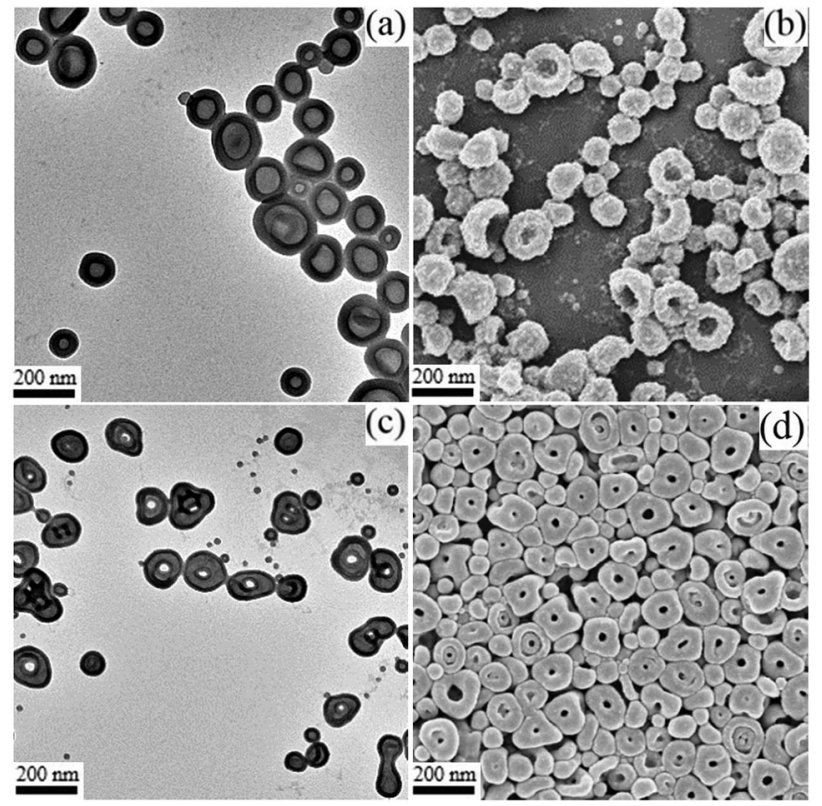

Fig. 4 TEM image of vesicles and toroidal vesicles at $[\mathrm{ANI}] /[\mathrm{SSA}]=0$ (a) and 0.4 (c), along with corresponding SEM image (b) and (d).

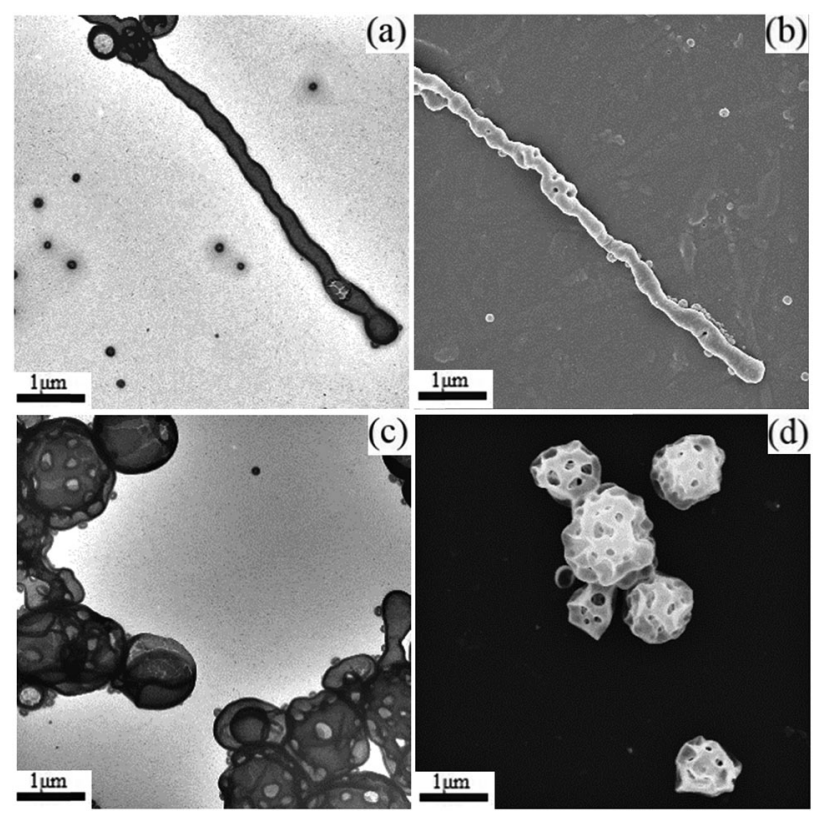

Fig. 5 TEM image of giant tubular vesicles and perforated spherical vesicles at $[\mathrm{ANI}] /[\mathrm{SSA}]=0.7$ (a) and 0.9 (c), along with corresponding SEM image (b) and (d).

Specifically, intercoronal repulsion due to negatively charged sulphonic acid groups in the core-corona interface decreases with increase in PANI concentration. Furthermore, the PS chain stretching may have decreased as a result of complexation with PANI and the overall increase in hydrophobic block content. ${ }^{23}$ The decrease in chain stretching of the bilayer forming block favors free energy minimization by eliminating the bending penalty associated with the formation of spherical vesicles. Combination of abovementioned factors may provide lower curvature effect to PANI

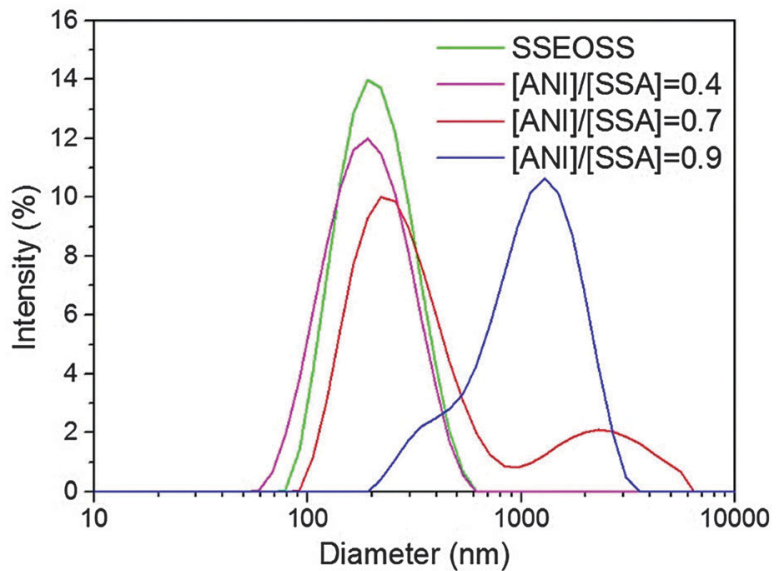

Fig. 6 Hydrodynamic diameter $\left(D_{h}\right)$ of aggregates from [PSS-PS]-PEO[PS-PSS]/PANI complexes at various PANI concentrations.

complexes during self-assembly. This leads to preferential segregation of PANI complexes towards toroidal exterior and free block copolymer chains towards toroidal interior. After introduction of PANI, at $[\mathrm{ANI}] /[\mathrm{SSA}]=0.4$, a decrease in $D_{\mathrm{h}}$ of aggregates to $207 \mathrm{~nm}$ (Fig. 6) was observed. The decrease in aggregate size can be ascribed to partial neutralization of sulphonic acid groups by PANI led to an overall reduction in the effective volume of hydrated sulphonic acid groups.

Further increasing PANI content to 0.7 led to the formation of giant tubular vesicles as seen in Fig. $5 \mathrm{a}$ and b. Tubular nanostructures from block copolymers and surfactants have been reported previously. ${ }^{24,25}$ However, giant tubules of diameter around $400 \mathrm{~nm}$ with an average length of $8-10 \mu \mathrm{m}$ were observed for the first time in this study from block copolymer complexes. It has been hypothesized that tubule formation was through fusion of spherical vesicles during the process of aggregation, confirmed via oscillatory perturbations observed in the diameter of the vesicles. ${ }^{24}$ The same phenomenon can be seen in these tubular structures. In addition, short tubes from fusion of multiple vesicles were observed above PANI content 0.4 and eventually lead to formation of long tubules at PANI content 0.7 (see ESI $\dagger$ ). The morphology transformation from toroidal vesicles to giant tubular vesicles, upon increasing PANI content, may be due to the crosslinking or bridging effect produced by stiff PANI chains as we have previously reported. ${ }^{19,26}$ As the PANI complexes reside in the core-corona interface, the bridging of sulfonic acid groups by PANI chains may have helped fusion of vesicular bilayers to form giant tubules. The entropic penalty associated with the chain packing frustration in the bilayer may have been overcome by strong interaction between sulfonic acid groups and PANI. In DLS measurement of non-spherical particles such as rods and tubes, the measured hydrodynamic diameter corresponds to the sphere that has same average translational diffusion coefficient produced by the particle under investigation. Here, the bimodal DLS peaks at $293 \mathrm{~nm}$ and $2.5 \mu \mathrm{m}$ distribution can be ascribed to the coexistence of spherical vesicles and tubules (Fig. 6). 


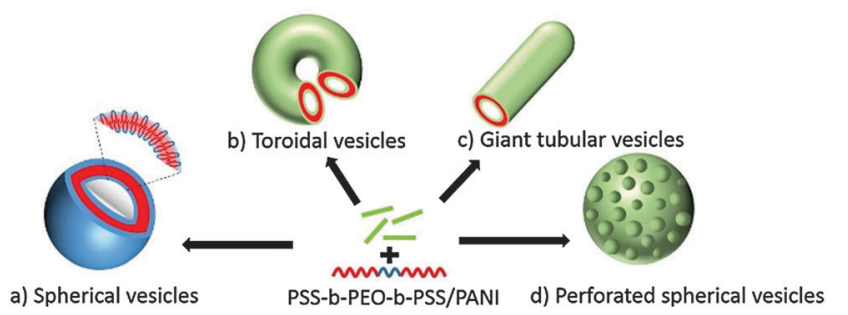

Fig. 7 Schematics of self-assembled aggregates of [PSS-PS]-PEO-[PSPSS]/PANI complexes at (a) [ANI]/[SSA] $=0$, (b) [ANI]/[SSA] $=0.4$, (c) [ANI]/ $[\mathrm{SSA}]=0.7$ and $(\mathrm{d})[\mathrm{ANI}] /[\mathrm{SSA}]=0.9$.

At $[\mathrm{ANI}] /[\mathrm{SSA}]=0.9$, perforated spherical vesicles were observed as shown in Fig. 5c and d. As described previously, at higher PANI concentrations, the lower curvature effect of PANI complexes facilitates formation of complex bilayer morphologies such as perforated spheres observed here. Similarly, nanostructures of complex morphologies have been prepared by varying the block copolymer-homopolymer compositions. ${ }^{27}$ We assume that the pores on the surface of giant vesicles may have been formed by the stalk-pore membrane fusion mechanism during the fusion of spherical vesicles. ${ }^{28,29}$ Furthermore, the internal segregation of free block copolymers with higher curvature effect may have formed the rim of the pores within the bilayer. The PANI cross-linking effect may have driven the membrane fusion in addition to the lower entropic penalty/surface tension associated with the formation of larger bilayer nanostructures. The hydrodynamic size of these perforated vesicles was around one $\mu \mathrm{m}$ as measured from DLS technique. Formation of smaller vesicles and PANI molecular aggregates may be responsible for the broad DLS peak at higher PANI contents. At PANI contents above 0.9, molecular aggregates of PANI led to macroscopic phase separation of block copolymer and PANI. Fig. 7 shows the schematic representation of the morphology transformation in PS-PEO-PS/PANI complexes as the function of varying PANI content.

We have reported new morphologies such as toroidal vesicles, giant tubular vesicles and perforated vesicles from selfassembled [PSS-PS]-PEO-[PS-PSS]/PANI complexes in aqueous solution. The morphology transformation was studied as the function of increasing PANI content. A possible mechanism for the morphology transformation upon increasing PANI content was also discussed. The rigidity and hydrophobic nature of PANI chains may be responsible for changing self-assembly parameters in flexible block copolymers even at its lower concentrations. Understanding the morphology evolution in bilayer nanostructures may enhance our knowledge of synthetic biology and towards achieving biomimicry.

A. P. was supported by Deakin University Postgraduate Research Scholarship (DUPRS).

\section{Notes and references}

1 J. Grumelard, A. Taubert and W. Meier, Chem. Commun., 2004, 1462-1463.

2 E. Yoshida, Colloid Polym. Sci., 2014, 292, 1463-1468.

3 M. Semsarilar, V. Ladmiral, A. Blanazs and S. P. Armes, Polym. Chem., 2014, 5, 3466-3475.

4 J. Zhu, S. Zhang, K. Zhang, X. Wang, J. W. Mays, K. L. Wooley and D. J. Pochan, Nat. Commun., 2013, 4, 2297.

5 M. Marguet, C. Bonduelle and S. Lecommandoux, Chem. Soc. Rev., 2013, 42, 512-529.

6 E. Yoshida, Colloid Polym. Sci., 2014, 292, 763-769.

7 Y. Matsushita, A. Noro and A. Takano, Polym. J., 2012, 44, 72-82.

8 A. Noro, K. Ishihara and Y. Matsushita, Macromolecules, 2011, 44, 6241-6244.

9 O. Ikkala and G. ten Brinke, Chem. Commun., 2004, 2131-2137.

10 J. Rolland, P. Guillet, J.-M. Schumers, N. Duhem, V. Préat and J.-F. Gohy, J. Polym. Sci., Part A: Polym. Chem., 2012, 50, 4484-4493.

11 N. Lefèvre, C.-A. Fustin and J.-F. Gohy, Macromol. Rapid Commun., 2009, 30, 1871-1888.

12 Z. Ge, J. Xu, D. Wu, R. Narain and S. Liu, Macromol. Chem. Phys., 2008, 209, 754-763.

13 N. V. Salim, T. L. Hanley, L. Waddington, P. G. Hartley and Q. Guo, Macromol. Rapid Commun., 2012, 33, 401-406.

14 A. O. Moughton, M. A. Hillmyer and T. P. Lodge, Macromolecules, $2011,45,2-19$.

15 C. V. Synatschke, T. Nomoto, H. Cabral, M. Förtsch, K. Toh, Y. Matsumoto, K. Miyazaki, A. Hanisch, F. H. Schacher, A. Kishimura, N. Nishiyama, A. H. E. Müller and K. Kataoka, ACS Nano, 2014, 8, 1161-1172.

16 L. Zhang and A. Eisenberg, Macromolecules, 1996, 29, 8805-8815.

17 G. Pasparakis, N. Krasnogor, L. Cronin, B. G. Davis and C. Alexander, Chem. Soc. Rev., 2010, 39, 286-300.

18 W. B. Stockton and M. F. Rubner, Macromolecules, 1997, 30, 2717-2725.

19 A. Palanisamy and Q. Guo, RSC Adv., 2014, 4, 54752-54759.

20 D. Mazzier, M. Mba, M. Zerbetto and A. Moretto, Chem. Commun., 2014, 50, 4571-4574.

21 H. Luo, J. L. Santos and M. Herrera-Alonso, Chem. Commun., 2014, 50, 536-538.

22 L. F. Zhang and A. Eisenberg, J. Am. Chem. Soc., 1996, 118, 3168-3181.

23 Y. Mai and A. Eisenberg, Chem. Soc. Rev., 2012, 41, 5969-5985.

24 Z. C. Li, Y. Shen, Y. Z. Liang and F. M. Li, Chin. J. Polym. Sci., 2001, 19, 297-301.

25 P. Yager and P. E. Schoen, Mol. Cryst. Liq. Cryst., 1984, 106, 371-381. 26 A. Palanisamy and Q. Guo, J. Phys. Chem. B, 2014, 118, 12796-12803.

27 D. V. Pergushov, A. H. E. Muller and F. H. Schacher, Chem. Soc. Rev., 2012, 41, 6888-6901.

28 M. Muller, K. Katsov and M. Schick, J. Polym. Sci., Part B: Polym. Phys., 2003, 41, 1441-1450.

29 G. J. A. Sevink and A. V. Zvelindovsky, Macromolecules, 2005, 38, $7502-7513$ 